\title{
Experimental verification of the properties of pneumatic elements
}

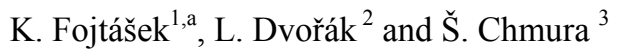 \\ ${ }^{1,2,3}$ VŠB-Technical University of Ostrava, Faculty of Mechanical Engineering, Department of Hydrodynamics \\ and Hydraulic Equipment, 17. listopadu 15/2172, Ostrava 70833, Czech Republic
}

\begin{abstract}
The subject of this article is experimental verification of properties of pneumatic elements. These elements have been a part of equipment for measuring of rotary air motors parameters. When comparing the measured data with the mathematical model, there were slight variations. The main objective is to verify the correct functioning of these pneumatic elements, comparing the measured results with theoretical data from the catalogs of manufacturers and determine their potential impact on the entire measuring system.
\end{abstract}

\section{Introduction}

Last year they were published two papers on the topic of measuring characteristics [1] and mathematical modeling of pneumatic rotary motors [2]. These publications included a description of the measurement and also the fact that one of the approaches to modeling did not show good results. This could be caused not only by the model of the rotary motor itself, but also influenced by elements of the measuring device used before the motor. For this reason, the characteristics of the basic elements used in the measuring circuit were measured to verify their correct functionality.

The subject of this article is the experimental verification of properties of pneumatic elements. When measuring it is necessary to comply with the general requirements for testing of pneumatic components. It is necessary to ensure a certain accuracy and credibility of the measured results as well as reproducibility and repeatability of each measurement. These general requirements are described in the ČSN 109006 standard for pneumatic elements. First of all, it is important to inspect the tested element properly, to ensure the inner and outer cleanliness of the element and its integrity. Check the functionality of the element and, if necessary, adjust the element according to the instructions or as specified in the technical specifications. The whole test circuit must then be checked for proper arrangement, functionality, leakage and used measuring equipment. The measuring equipment must have the correct measuring ranges and the correct accuracy class. The whole circuit must be built according to a predetermined scheme. The elements are mounted in the prescribed position and emphasis must be placed on safety. All measuring devices and sensors must undergo inspection or calibration prior to the measurement. If they change their parameters as a result of use during the test, they

\footnotetext{
${ }^{\mathrm{a}}$ Corresponding author: kamil.fojtasek@vsb.cz
}

must be checked again after the test. If a deviation is greater than permitted class of precision the measurements must be repeated. It is also necessary that each measurement be carried out in a steady state unless there are special requirements for the dynamics of the measured element and the measurements must be made at least three times. All measured values are recorded during the measurement, test conditions (ambient temperature, air pollution class, atmospheric pressure, vibration, impact, etc.), date, place of measurement, data on measurement methodology and measuring devices and also the test system scheme. These data are based on test report, which contains the main technical parameters of the test element and their comparison with the measurement results. The measurement results are displayed either in the table or in the graph as functional dependencies [3].

\section{Theory of measurement}

Measured elements are a throttling valve, a pressure reducing valve and a directional control valve whose measurement methodology is described in ISO 6358 standard. This is sonic conductance measurements, critical pressure ratio and normal nominal flow rate. Another alternative is measurement according to the Japanese standard JIS B 8390, where the effective area (equivalent cross-section) is measured.

According to the International Standard, the flow characteristics are given as a result of the comparison between the sonic conductance $C\left[d \mathrm{~m}^{3} \cdot \mathrm{s}^{-1} \cdot \mathrm{bar}^{-1}\right]$ and the critical pressure ratio $b[-]$. Sonic conductance $C$ is the maximum flow capacity of any pneumatic element. Maximum capacity is reached when the passing gas reaches the choked flow. The critical pressure ratio $b$ 
defines the pressure ratio at which the flow in the element is changing Fig. 1.

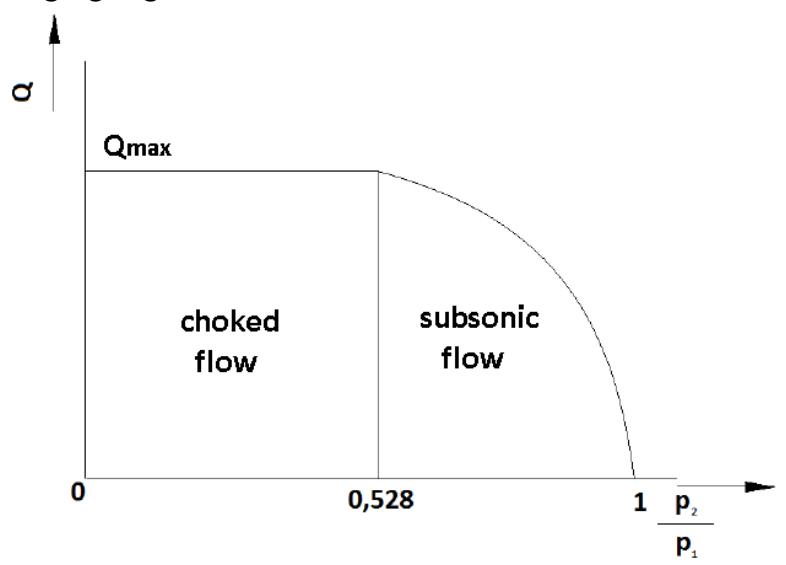

Fig. 1. Types of flow

At throttled flow, the input (upstream) pressure is higher than the output (downstream) pressure on the measured element, and at some part of the element the sound speed is reached. The standard defines the basic conditions, air temperature $20^{\circ} \mathrm{C}$, absolute pressure $1 \mathrm{bar}$ and relative humidity $65 \%$. To calculate the flow rate through the measured element, if $\frac{p_{2}+1}{p_{1}+1} \leq b$ (choked flow)

$$
Q=60 \cdot C\left(p_{1}+1\right) \sqrt{\frac{293}{273+t}}
$$

For subsonic flow, if $\frac{p_{2}+1}{p_{1}+1}>b$ is valid

$$
Q=60 \cdot C\left(p_{1}+1\right) \sqrt{\left[\frac{p_{2}+1}{p_{1}+1} \cdot b\right]^{2}} \sqrt{\frac{293}{273+t}}
$$

Figure 2 shows a measuring circuit according to ISO 6358 standard. By attaching a test equipment with the test circuit, the process of measurement and determination of parameters are follows. Upstream pressure $p_{1}$ have to be minimally 3 bar and all the time of measurement have to be constant. Throttling valve is fully open. Now maximal (sonic) flow rate $Q_{m}^{*}$ can be measured. Sonic conductance $C$ can be determine by formula

$$
C=\frac{Q_{m}^{*}}{\rho_{0} \cdot p_{1}} \sqrt{\frac{T_{1}^{*}}{T_{0}}}
$$

Calculation of pressure ratio $b$ requires measured pressure $p_{1}, p_{2}$, temperature $T_{1}$ and flow rate by the four other settings of flow rate $Q_{m}$ yet $(80 \%, 60 \%, 40 \%$ and $20 \%$ of sonic flow $Q_{m}^{*}$ ). Flow can be changed by throttling valve. Critical pressure ratio $b$ is determined as a mean value of pressure ratios $b$ for each of flow rate $Q_{m}$. Single pressure ratios can be calculated by formula

$$
b=1-\frac{\frac{\Delta p}{p_{1}}}{1-\sqrt{1-\left(\frac{Q_{m}}{Q_{m}^{*}}\right)^{2}}}
$$

On the same track, the normal nominal flow rate is measured at the inlet pressure of 6 bar and 5 bar at the outlet of the element.

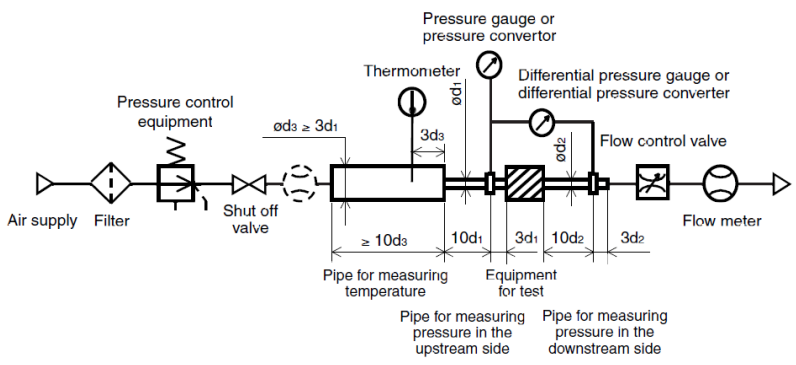

Fig. 2. Measuring circuit according to ISO 6358 standard [4]

JIS B 8390 standard defines another measurement method. Describe the effective area $S$, which is the crosssectional area having an ideal throttle without friction deduced from the calculation of the pressure changes inside an air tank or without reduced flow when discharging the compressed air in a choked flow, from an equipment attached to the air tank. Again according to the type of the flow, two basic equations can be determined.

For choked flow $\frac{p_{2}+1}{p_{1}+1} \leq 0.5$ is flow rate

$$
Q=12 \cdot S\left(p_{1}+1\right) \sqrt{\frac{293}{273+t}}
$$

For subsonic flow $\frac{p_{2}+1}{p_{1}+1}>0.5$

$$
Q=24 \cdot S \sqrt{\left(p_{2}+1\right)\left(p_{1}-p_{2}\right)} \sqrt{\frac{293}{273+t}}
$$

Measuring circuit according to JIS B 8390 standard is in Fig. 3. The air tank must be pressurized to a certain pressure which must not fall below 6 bar. When compressed air is discharged, the time at which the pressure in the air tank drops to 2.5 bar is measured.

Now it is also possible to calculate the effective area $\mathrm{S}$ according to the equation (7). The volume of the air tank shall be selected within a given range corresponding to the relative cross-section of the test element.

$$
S=1.21 \frac{V}{t} \log _{10}\left(\frac{p_{s}+1}{p+1}\right) \sqrt{\frac{293}{T}}
$$

$S$ is effective area $\left[\mathrm{mm}^{2}\right], V$ is air tank capacity $\left[\mathrm{dm}^{3}\right], t$ is discharging time $[s], p_{s}$ is pressure inside air tank before discharging [bar], $p$ is residual pressure inside air tank after discharging [bar] and $T$ is temperature inside air tank before discharging $[T]$.

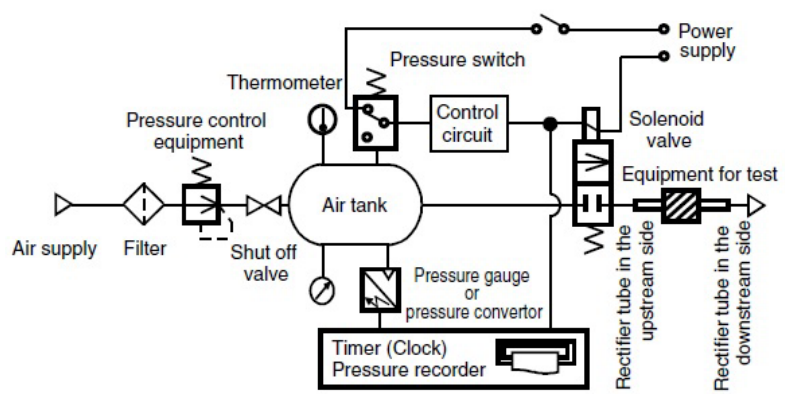

Fig. 3. Measuring circuit according to JIS B 8390 standard [4] 


\section{Measurement methodology}

The design of the track for measuring the flow characteristics of the throttling and pressure reducing valve was based on ISO 6358 standard. Measured values was inlet pressure $p_{1}$, outlet pressure $p_{2}$ (before and behind the measured element), temperature $T$ and flow rate $Q$. The temperature was measured at the beginning of the measuring track, the flow meter was positioned at the end of the track with respect to its type. The scheme of the measuring track is in Fig. 4.

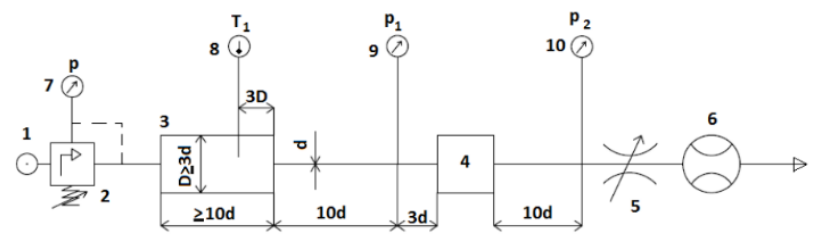

Fig. 4. Scheme of the measuring track for throttling and pressure reducing valves

1 - source of compressed air, 2 - pressure reducing valve,

3 -air tank, 4-measured element, 5 -throttling valve,

6 -flow meter, 7 -control manometer, 8 - thermometer,

9 - manometer for inlet pressure measurement, 10 - manometer for outlet pressure measurement, $d$-inner diameter of the pipe, $D$ - diameter of air tank

In the case of the measurement of the throttling valve, after the source of compressed air 1 , a reducing valve 2 is located which adjusts and regulates the constant pressure $p_{1}$ at the inlet of the measured element 4 . The throttling valve 5 is completely closed at the beginning of the measurement. Turn the measured throttling valve 4 to a certain number of revolutions and start measuring. By gradual opening of the throttling valve 5 by certain revolutions, the outlet pressure $p_{2}$ and the flow rate $Q$ (measured by the flow meter 6) are also increasing. Measurement is shown in Fig. 5.

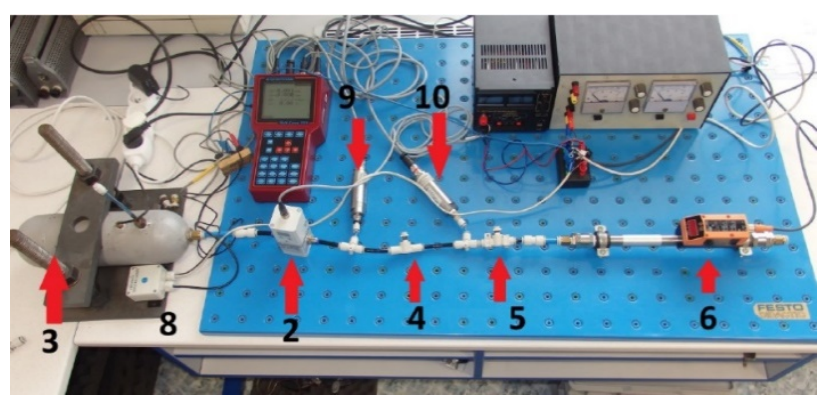

Fig. 5. Realization of throttling valve measuring

2 - reducing valve, 3 - air tank, 4-measured throttling valve, 5 -throttling valve, 6 -flow meter, 8 - thermometer, 9 -inlet pressure sensor, 10 - outlet pressure sensor

The measured element was a throttling valve AS $3001 \mathrm{~F}$. The working range of the valve is $1 \div$ 10 bar. The operating temperature is $-5 \div 60^{\circ} \mathrm{C}$ and nominal size is $10 \mathrm{~mm}$. The throttle valve was measured for 4 different revolutions settings and set to $5.5 \mathrm{rev}$, 6.5 rev, 8 and 10 revolutions.

The measuring track for the reduction valve is the same as in Fig. 4. Behind the source of compressed air 1 is a reduction valve 2 , which sets the constant pressure $p_{1}$ at the inlet of the masured valve 4 . On the pressure valve 4 we set the same pressure like at the inlet or the less pressure. The measurement is carried out by gradually opening the throttle valve 5, thereby changing the pressure and flow, at the same time the inlet pressure must be controlled by the pressure regulator 2 . The flow rate and temperature is measured as in the previous measurement. Realization of measurement is shown in Fig. 6.

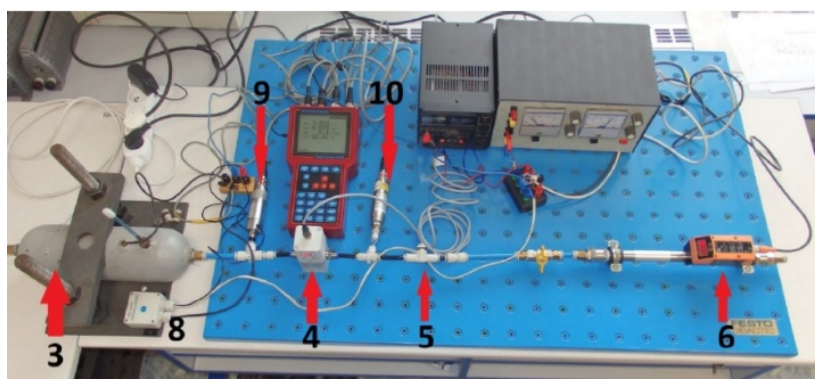

Fig. 6. Realization of reducing valve measuring 3 -air tank , 4-measured reducing valve, 5-throttling valve, 6 -flow meter, 8 -thermometer, 9 -imput pressure sensor, 10 output pressure sensor

The measured element was a pressure reducing valve VPPE-3-1-1/8-10-010-E1 Festo. The control range of this valve is $0.1 \div 10 \mathrm{bar}$ and is capable of operating at ambient temperature $0 \div 60^{\circ} \mathrm{C}$ and the temperature of the medium $10 \div 50^{\circ} \mathrm{C}$. The reducing valve was measured for 4 different settings 3, 4, 5 and 6 bar.

The scheme of the measuring track for measuring the $5 / 2$ directional valve is shown in Fig. 7. Behind the source of compressed air 1 , is a reducing valve 2 to adjust the inlet pressure to the directional valve 6 . Shut off valve 5 open to full flow and measure the discharge time of the air tanks 3 and 4 . Connection is made only in one direction of the valve, return strand is blinded. During the measurement, the directional valve is not switching. The layout of the elements on the scheme is based on the JIS B $8390 \quad$ standard.

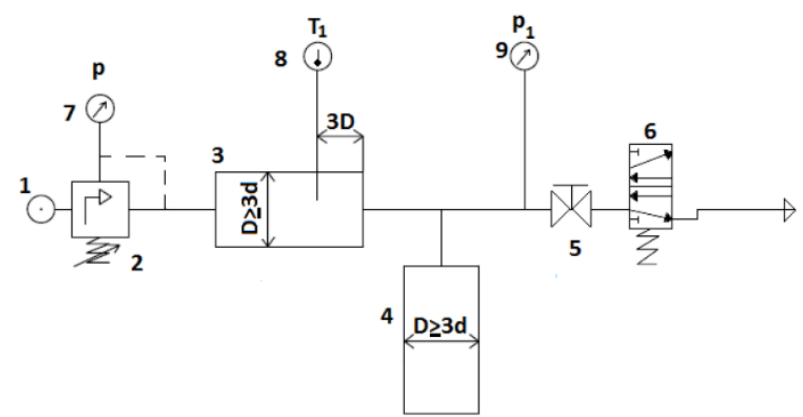

Fig. 7. Scheme of the measuring track for directional valve 1 - source of compressed air, 2 - pressure reducing valve, 3 -air tank , 4-air tank, 5-shut off valve, 6-measured directional valve, 7 - control manometer, 8 - thermometer, 9 - manometer for inlet pressure measurement

Measured directional valve was 5/2 SMC SYA3120 M5. The working range of the valve is $1.5 \div 7 \mathrm{bar}$ and is capable of operating at ambient and medium temperature $-10 \div 60^{\circ} \mathrm{C}$. Nominal flow rate is $171 \mathrm{dm}^{3} \cdot \mathrm{min}^{-1}$. Realization of measuring is shown in Fig. 8.

Overall, there were three measurements. The first measurement can only be considered as a test. The remaining two measurements can be considered as final. During the measuring were recorded time values of 
discharging of air tank. These values were measured until to pressure 2.5 bar. Then, according to equation (7) was calculate effective area $S$.

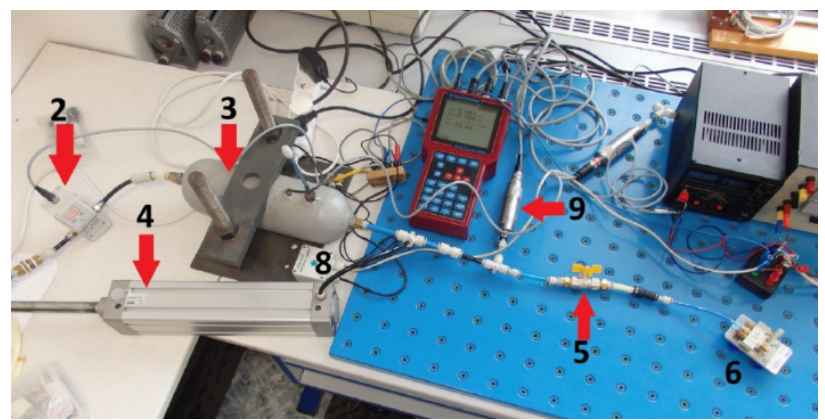

Fig. 8. Realization of directional valve measuring

2 - reduction valve, 3,4 air tank, 5- shut off valve, 6 - measured directional valve, 8 - thermometer, 9 -inlet pressure sensor

\section{Conclusion}

This chapter lists the measurement results and the conclusions that follow. In the case of a throttling valve, the manufacturer does not specify the characteristics of this valve in the catalog, so it cannot be compared with it. However, according to the visual comparison with the theoretical characteristics of the throttle valves, it can be assumed that the throttle valve has the correct flow throughput at the given setting. The resulting measured flow characteristic is shown in Fig. 9.

In the case of a reduction valve, the measured characteristics are consistent with the characteristics stated by the manufacturer. Due to the conditions in the pneumatic mechanism laboratory, it was not possible to verify the properties of the reduction valve over the entire flow range. However, this was not necessary with respect to flow rates when measuring rotary motors. Measurements at a pressure of $p_{1}=6 \mathrm{bar}$ were influenced by the system before the measuring line and lack of permeability. The resulting characteristic with its course coincides with the characteristics of the manufacturer and their comparison is shown in Fig. 10.

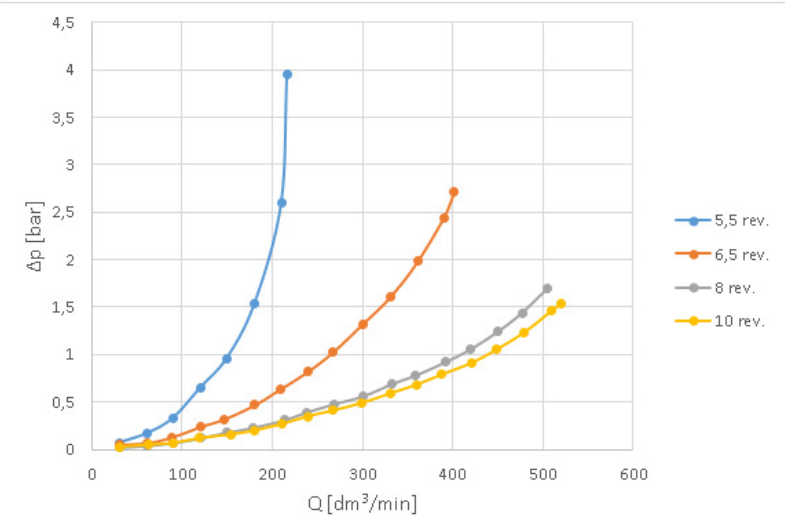

Fig. 9. Measured flow characteristic of throttling valve [5]

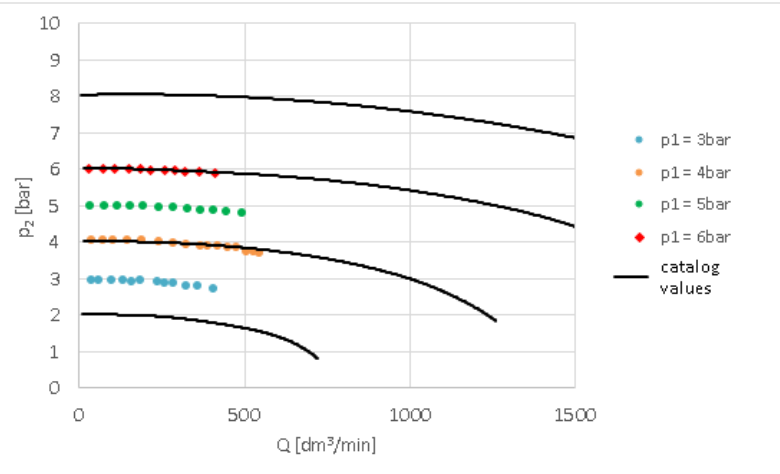

Fig. 10. Comparison of the measured characteristics of the reduction valve with the information given in the manufacturer's catalog [5]

For directional valves was determined effective area $S$ by the measured values of discharging air tank. Its average value for all measurements was $S=2.619 \mathrm{~mm}^{2}$. Subsequently, for comparison with catalog data, the effective area was recalculated to the $C_{v}$ factor. Resulting values of this factor was 0.144 , which is lower than the value stated in the manufacturer's catalog $(0.16)$. This deviation could be due to the wear of the measured element or insufficient volume of the air tank. To confirm this statement would have to be carried out another series of measurements, with a longer discharging time of air tank.

The measured flow characteristics of the pneumatic elements will be further used to compare and modify the mathematical model of the pneumatic system with the rotary pneumatic motor. Based on the measurements, it was confirmed correct operation of the throttling and a pressure reducing valve. For subsequent control measurement of rotary air motors, will be replaced directional valve by a new element.

\section{Acknowledgements}

The work presented in this paper was supported by a grant SGS „Research in field of fluid system dynamics" SP2017/103.

\section{References}

1. L. Dvořák, K. Fojtášek, V. Řeháček, Experimental Equipment for Measuring of Rotary Air Motors Parameters, EPJ Web of Conferences, Volume 143 (2017)

2. L. Dvořák, K. Fojtášek, V. Řeháček, Calculations of Parameters and Mathematical Model of Rotary air Motor, EPJ Web of Conferences, Volume 143 (2017)

3. J. Kopáček, Pneumatické mechanismy (1998)

4. SMC, Solenoid Valves Flow Characteristics (1998)

5. Š. Chmura, Measurement of Characteristics of Pneumatic Components, Bachelor Thesis (2017) 